\title{
Efecto biocida del «barbasco» Lonchocarpus utilis (Smith,1930) como regulador de larvas de mosquitos
}

\section{Biocidal effect of «barbasco» Lonchocarpus utilis $($ Smith, 1930) as regulator of mosquitoes larvae}

\author{
Carlos Mariños ${ }^{1}$, Julia Castro ${ }^{2}$ y Diana Nongrados ${ }^{2}$
}

Presentado: $11 / 05 / 2004$

Aceptado: $\quad 12 / 07 / 2004$

\section{Resumen}

Se realizaron 7 bioensayos de laboratorio para evaluar la capacidad biocida de Lonchocarpus utilis (Smith,1930) «barbasco» sobre 7000 larvas de tercer y cuarto estadio de Anopheles benarrochi (Gabaldón, 1941), vector primario de malaria, en Yurimaguas y Loreto. La actividad biocida fue determinada con diferentes calidades de agua. Se procesaron las raíces frescas de $L$. utilis siguiendo el procedimiento utilizado por Vílchez (1993), obteniéndose como producto final un polvo fino que contiene como principio activo la rotenona. La actividad biocida, se midió con 5 dosis de polvo de la raíz diluida en agua destilada: 6,$25 ; 3,1 ; 2,1 ; 1,0$ y $0,15 \mathrm{~g} / \mathrm{L}$. Se utilizó $1 \mathrm{~mL}$ del homogenizado como inóculo por dosis. Para determinar la eficacia y susceptibilidad se realizaron lecturas cada hora hasta las 24 horas después del tratamiento. A las 12 horas postratamiento, las dosis de 6,25 y $3,1 \mathrm{~g} / \mathrm{L}$, mostraron 98 y $89 \%$ de mortalidad larvaria cuando se utilizó agua destilada y $86 \%$ y $82 \%$ cuando se utilizó agua de criadero. A las 24 horas la mortalidad alcanzó el 99 y $94 \%$ usando agua destilada y con agua de criadero fue 93 y $90 \%$. A las 6 horas de exposición con agua destilada, la dosis letal media (DL50) fue de 0,63 g/L y la dosis letal noventa (DL90) fue de 12,44 g/L; mientras a las 12 horas la DL50 fue de $0,48 \mathrm{~g} / \mathrm{L}$ y la DL90 7,23 g/L. Utilizando agua de criadero a las 6 horas la DL50 fue de 1,36 g/L y la DL90 fue de 27,58 g/L; mientras que a las 12 horas la DL50 fue de 0,83 g/L y la DL90 fue de 9,83 g/L del extracto crudo de $L$ utilis. Los resultados permitieron comprobar la efectividad del polvo de raíz de Lonchocarpus utilis sobre larvas de $A$. benarrochi como potencial biocida y que su acción está influenciada por la calidad del agua y la dosis de aplicación.

Palabras clave: Lonchocarpus utilis, Anopheles benarrochi, actividad biocida, larvicida.

\section{Abstract}

Seven bioassays in laboratory were carried out in order to evaluate the biocidal capacity of Lonchocarpus utilis (Smith,1930) «barbasco» on 7000 larvae of third and fourth stage of Anopheles benarrochi (Gabaldon,1941), main vector of malaria in Yurimaguas and Loreto. The biocidal activity was probed with two types of quality water. Fresh roots of $L$. utilis were processed by Vílchez (1993) methodology, it was obtained a fined powder which contains as active ingredient the rotenone. The biocidal activity was measured with 5 doses of powder of the root diluted in distilled water: 6,$25 ; 3,1 ; 2,1$ ; 1,0 and $0,15 \mathrm{~g} / \mathrm{L}$. It was used $1 \mathrm{~mL}$ of the homogenized as inoculum for dose. Readings were made hourly until the 24 hours post treatment to determine the effectiveness and susceptibility. At the 12 hours post-treatment the doses of 6,25 and $3,1 \mathrm{~g} / \mathrm{L}$, obtained 98 and $89 \%$ of larval mortality when it was utilized in distilled water and in water of breeding site mortality was of 86 and $82 \%$ respectively. At the 24 hours mortality reached 99 and $94 \%$ using distilled water and with water of breeding site was 93 and $90 \%$. At the 6 hours of exposure with distilled water, the medium lethal dose (DL50) was of 0,63 g/ $L$ and the lethal dose ninety (DL90) it was of $12,44 \mathrm{~g} / \mathrm{L}$; while at the 12 hours the DL50 was of $0,48 \mathrm{~g} /$ $\mathrm{L}$ and the DL90 was $7,23 \mathrm{~g} / \mathrm{L}$. Utilizing breeding site water at the 6 hours, the DL50 was of $1,36 \mathrm{~g} / \mathrm{L}$ and the DL90 it was $27,58 \mathrm{~g} / \mathrm{L}$; while at the 12 hours the DL50 was $0,83 \mathrm{~g} / \mathrm{L}$ and DL90 it was of $9,83 \mathrm{~g} / \mathrm{L}$ of the extract raw of $L$. utilis on the larvaes. The results made it possible to verify the effectiveness of the raw extract of $L$. utilis on larvae of $A$. benarrochi as potential larvicide and its action is influenced by the water quality and the dose of application.

Keywords: Lonchocarpus utilis, Anopheles benarrochi, biocid activity, larvicide.

${ }^{1}$ Laboratorio de Entomología, Facultad de Zootecnia, Universidad Nacional de la Amazonía, Perú

${ }^{2}$ Laboratorio de Inmunología Parasitaria. Facultad de Ciencias Biológicas Universidad Nacional Mayor de San

Marcos. Apartado Postal 11-0058, Lima 11, Perú. Email Julia Castro:jcastroh@unmsm.edu.pe 


\section{Introducción}

A pesar de los esfuerzos por controlar la malaria el número de casos se han venido incrementando porque al igual que otras enfermedades metaxénicas esta relacionada con las condiciones de pobreza y es en las personas de menores recursos donde su impacto es mayor (Proyecto Vigia, 1999).

Los insecticidas químicos han sido las principales herramientas en la estrategia para controlar los vectores de la malaria en el mundo, pero han sido demostrados los efectos nocivos para la salud y el medio ambiente sumados a la aparición de insectos resistentes y el efecto letal sobre organismos benéficos (RAAA, 1993). En países como el nuestro, se presentan problemas adicionales como el alto costo de los insecticidas sintéticos, los suministros erráticos, la falta de materiales y de equipos de aplicación además de un conocimiento adecuado sobre su uso, agravando de esta manera la lucha antivectorial.

A la falta de recursos económicos, en nuestro país hay que agregar la dificultad de acceso por falta de medios de transporte, los que imposibilitan la aplicación el control de brotes epidémicos de manera oportuna.

Estos antecedentes motivan la búsqueda de métodos alternativos seguros y eficaces como las plantas con propiedades biocidas entre las que se encuentra Lonchocarpus utilis (Smith, 1930) o «barbasco» cuyo princi-

Tabla 1. Características físico-químicas del agua de criadero utilizada en los bioensayos de laboratorio

\begin{tabular}{lc}
\hline Indicadores & $\begin{array}{c}\text { Agua de Criadero } \\
\text { de } \text { Anopheles benarrochi }\end{array}$ \\
\hline $\mathrm{pH}$ & 4,9 \\
Temperatura ${ }^{\circ} \mathrm{C}$ & $25^{\circ} \mathrm{C}$ \\
Turbiedad & $2,01 \mathrm{UNT}$ \\
Color & $25 \mathrm{Unidad} \mathrm{Color}$ \\
Cloruros & $9 \mathrm{mg} / \mathrm{L} \mathrm{Cl}-1$ \\
Sólidos totales disueltos & $356 \mathrm{mg} / \mathrm{L}$ \\
Nitritos & $0,08 \mathrm{ppm}$ \\
OD ppm & $2,8 \mathrm{ppm}$ \\
Conductividad & $329 \mathrm{umhos} / \mathrm{cm}$ \\
NMP coliformes totales & $5,3 \times 10^{4} \mathrm{UFC} / \mathrm{mL}$ \\
NMP coliformes fecales & $1 \times 10^{2} \mathrm{UFC} / \mathrm{mL}$ \\
\hline
\end{tabular}

pio activo es la rotenona. Estas plantas constituyen fábricas naturales de plaguicidas botánicos con diversas propiedades biológicas.

En el Perú, la aparición de insecticidas sintéticos alejó a la rotenona de la actividad agrícola como insecticida, hasta ser casi olvidada por las nuevas generaciones de agricultores, a pesar de que la rotenona es un producto de exportación cuyo uso está regulado por organismos internacionales encargados de supervisar la toxicidad de los insecticidas (Lizárraga, 1993).

Se espera que el uso de productos alternativos autóctonos logrará reducir la importación de insecticidas y disponer de una herramienta que permita de manera oportuna actuar frente a la aparición de brotes epidémicos. Entonces es necesario retomar esta experiencia y tecnología para volver a utilizar este insecticida casi olvidado, por sus características de ser potente, de muy corto poder residual, baja toxicidad para el hombre y los animales de sangre caliente y sobre todo muy barato y compatible para el control de plagas en las diferentes zonas del Perú (CDPI-CIP, 1992).

El presente estudio evalúa en bioensayos de laboratorio la actividad biocida del extracto crudo de L. utilis sobre larvas de A. (Nys) benarrochi, vector de Plasmodium vivax y Plasmodium falciparum en la llanura amazónica del Perú.

\section{Material y métodos}

\section{Identificación de criaderos}

Se obtuvieron larvas de Anopheles benarrochi de criaderos naturales de la localidad de Munichis, distante $30 \mathrm{~km}$ del distrito de Yurimaguas, provincia Alto Amazonas, departamento de Loreto. Se evaluaron los parámetros físico-químicos del agua de los criaderos utilizados en los bioensayos y para el mantenimiento de larvas en el laboratorio (Tabla 1).

\section{Mantenimiento de la población blanco en condiciones de laboratorio}

Se seleccionaron los individuos de 3 er y 4to estadio de la misma especie y se colocaron para su observación por 2 días, en bande- 
jas de plástico con agua de criadero a temperatura ambiente y alimentadas con pellet pulverizado para aves hasta el momento en que se realizaron los bioensayos (Nongrados, 1999). Para determinar la especie de mosquito existente en los criaderos muestreados, se utilizaron las claves entomológicas para adultos y estadíos larvarios de Consoli (1994); Calderón et al. (1995) y Gorham et al. (1973).

\section{Obtención del extracto crudo}

Las plantas de «barbasco» fueron obtenidas en las inmediaciones del distrito de Jeberos, ubicado aproximadamente a 15 minutos de Yurimaguas por vía aérea. En esta zona se cultivan los «barbascales» asociados a cultivos de yuca y piña. La identificación taxonómica de la planta como Lonchocarpus utilis, fue realizada en el Museo de Historia Natural de la Universidad nacional Mayor de San Marcos (Herbario USM).

L. utilis (Smith, 1930) «barbasco» ó «cube», es una planta arbustiva leguminosa de la Familia Papilionaceae, que se encuentra en toda la Amazonía, cuyo principio activo es la rotenona catalogado como insecticida tipo II (OMS, 1988).

Las raíces frescas en secciones de $2 \mathrm{~cm}$ de diámetro, se picaron y se sometieron a deshidratación a $50{ }^{\circ} \mathrm{C}$ por 48 horas. Luego las raíces secas fueron trituradas en un molino de mano y tamizadas por un colador de malla fina, obteniéndose como producto final un polvo fino que fue guardado en frascos herméticamente cerrados y mantenidos en lugar seco y sombreado (Vílchez, 1993). En los bioensayos se utilizó como formulación del biocida, el polvo de la raíz en agua destilada.

\section{Preparación de los inóculos}

Se tomó como dosis de referencia la utilizada por Gamarra (1940) de 25 y 10 g/L del polvo de raíz diluido de $L$. utilis en series de proporciones media y logarítmica, probadas previamente. Se prepararon 5 dosis pesándose 6,$25 ; 3,1$; 2,$1 ; 1,0$ y $0,15 \mathrm{~g}$ del polvo de la raíz de L. utilis y diluidas en un litro de agua destilada, se homogenizaron y se tamizaron obteniéndose las dosis finales de 6,$25 ; 3,1 ; 2,1 ; 1,0$ y 0,15 g/L. En los bioensayos se utilizó un inóculo de $1 \mathrm{~mL}$ de cada una de las dosis preparadas previamente.

Cada dosis del biocida fue probada por triplicado, contra 25 larvas de $A$. benarrochi, utilizándose en total 75 larvas por dosis y 25 larvas para los controles por cada dosis. En total se utilizaron 500 larvas con agua destilada y 500 con agua de criadero, sumando un total de 1000 larvas por réplica.

\section{Eficacia y susceptibilidad de $A$. benarrochi frente a $L$. utilis}

Se realizaron 7 réplicas para determinar la susceptibilidad y eficacia del polvo de la raíz de L. utilis sobre $A$. benarrochi. Los bioensayos se realizaron en vasos de plástico de $7,5 \mathrm{~cm}$ de ancho por $8,5 \mathrm{~cm}$ de alto. Cuatro vasos fueron usados por dosis, tres para la prueba de biocida y uno para el control de la prueba. A cada vaso se le agregó $30 \mathrm{~mL}$ de agua destilada o de criadero y con una pipeta se trasladaron lotes de 25 larvas de $A$. benarrochi por vaso. Seguidamente cada vaso se completó hasta un volumen total de $150 \mathrm{~mL}$ lo cual corresponde al volumen donde se encuentran contenidas las larvas en estudio. Se anotaron la temperatura media del agua y el $\mathrm{pH}$, durante cada réplica siguiendo las recomendaciones de la OMS $(1960,1976)$. El vaso control se preparó solo con agua destilada o de criadero y 25 larvas.

El recuento de la mortalidad larvaria post tratamiento se hizo cada hora, durante las primeras 12 horas y a las 24 horas. Los porcentajes de mortalidad de cada concentración se calcularon con la cifra total de larvas muertas por vaso. La susceptibilidad se reportó como dosis letal media (DL50) y dosis letal noventa (DL90), utilizando el programa EPA probit 1.4 (Tabla 2).

\section{Análisis estadístico}

Para determinar la significancía de la mortalidad de larvas de $A$. benarrochi por efecto del polvo de raíz de L. utilis en ambos tipos de agua y entre las dosis utilizadas se aplicó la prueba no paramétrica de Kruskal-Wallis. Para medir si la calidad del agua interviene en las 
Tabla 2. Protocolo de los bioensayos. Número de larvas de Anopheles benarrochi por prueba.

\begin{tabular}{|c|c|c|c|c|c|}
\hline \multirow{2}{*}{$\begin{array}{l}\text { Concentración } \\
\text { del extracto } \\
\text { crudo de } L . \text { utilis } \\
(\mathrm{g} / \mathrm{L})\end{array}$} & \multicolumn{2}{|c|}{ Agua destilada } & \multicolumn{2}{|c|}{ Agua criadero } & \multirow{2}{*}{$\begin{array}{l}\text { Total de } \\
\text { larvas por } \\
\text { dosis de } \\
\text { aplicación }\end{array}$} \\
\hline & Testigo & Control & Testigo & Control & \\
\hline 0,15 & 75 & 25 & 75 & 25 & 200 \\
\hline 1 & 75 & 25 & 75 & 25 & 200 \\
\hline 2,1 & 75 & 25 & 75 & 25 & 200 \\
\hline 3,1 & 75 & 25 & 75 & 25 & 200 \\
\hline 6,25 & 75 & 25 & 75 & 25 & 200 \\
\hline $\begin{array}{l}\text { Total de larvas } \\
\text { por bioensayo }\end{array}$ & 375 & 125 & 375 & 125 & $\begin{array}{c}1000 \times 7= \\
7000\end{array}$ \\
\hline
\end{tabular}

pruebas de susceptibilidad, se empleó el método de análisis multivariado (MANOVA) del paquete estadístico SPSS versión WIN 7,5. Las variables a usar para sustentar este análisis fueron las dosis en gr/L de polvo de raíz de L. utilis y el tipo de agua (agua destilada y agua de criadero).

\section{Resultados}

\section{Bioensayos de laboratorio}

Los resultados de la eficacia del polvo de raíz de L. utilis, como factor que determina la mortalidad de las larvas de $A$. benarrochi durante los bioensayos, son presentados en las Tablas 3 y 4; donde los valores máximos de mortalidad se obtuvieron entre las 12 horas y 24 horas post-tratamiento en ambos tipos de agua (agua destilada y agua de criadero). Antes de medir la los resultados del tratamiento se realizó la lectura en los vasos controles.

Tabla 3. Porcentaje de mortalidad larvaria de Anopheles benarrochi en agua destilada por acción del polvo de raíz de Lonchocarpus utilis.

\begin{tabular}{cccccc}
\hline & \multicolumn{5}{c}{ Dosis de exposición $(\mathbf{g} / \mathrm{L})$} \\
Tiempo & $\mathbf{6 , 2 5}$ & $\mathbf{3 , 1}$ & $\mathbf{2 . 1}$ & $\mathbf{1}$ & $\mathbf{0 , 1 5}$ \\
\hline 0 & 0 & 0 & 0 & 0 & 0 \\
1 & 45 & 45 & 34 & 9 & 4 \\
2 & 66 & 61 & 50 & 22 & 12 \\
3 & 75 & 68 & 54 & 28 & 24 \\
4 & 82 & 73 & 59 & 35 & 28 \\
5 & 87 & 77 & 60 & 36 & 35 \\
6 & 91 & 81 & 62 & 36 & 36 \\
7 & 94 & 81 & 64 & 39 & 36 \\
8 & 94 & 82 & 65 & 40 & 38 \\
9 & 95 & 84 & 66 & 41 & 41 \\
10 & 96 & 84 & 67 & 41 & 41 \\
11 & 97 & 86 & 69 & 41 & 41 \\
12 & 98 & 89 & 71 & 41 & 41 \\
24 & 99 & 94 & 73 & 41 & 41 \\
\hline
\end{tabular}

Los tratamientos con las dosis 6,25 y $3,1 \mathrm{~g} /$ L después de 12 horas de inoculación, presentaron mayores valores de mortalidad, en agua destilada (98 y 89\%) con respecto a agua de criadero (86 y $82 \%$ ). A las 24 horas los valores de mortalidad fueron 99 y $94 \%$ en agua destilada y 93 y $90 \%$ en agua de criadero.

Asimismo en agua destilada, con las dosis 6,25 y $3,1 \mathrm{~g} / \mathrm{L}$ se observó mortalidades superiores al $60 \%$ a las 2 horas post-aplicación, mientras que con la dosis $2,1 \mathrm{~g} / \mathrm{L}$ la mortalidad fue mayor al $50 \%$ a las 3 horas post-aplicación. Con las dosis de 1,0 y $0,1 \mathrm{~g} / \mathrm{L}$ no se lograron de mortalidades superiores al 50\% del total de larvas durante el tiempo de desarrollo de los bioensayos, presentando estabilización en los porcentajes de mortalidad posterior a las 7 horas con ambas dosis.

Tabla 4. Porcentaje de mortalidad larvaria de Anopheles benarrochi en agua de criadero por accióndel polvo de raíz de Lonchocarpus utilis.

\begin{tabular}{cccccc}
\hline \multicolumn{7}{c}{ Dosis de exposición } \\
Tiempo & $\mathbf{6 , 2 5}$ & $\mathbf{3 , 1}$ & $\mathbf{2 , 1}$ & $\mathbf{1}$ & $\mathbf{0 , 1 5}$ \\
\hline 0 & 0 & 0 & 0 & 0 & 0 \\
1 & 31 & 21 & 25 & 7 & 10 \\
2 & 56 & 45 & 35 & 20 & 12 \\
3 & 66 & 56 & 42 & 22 & 15 \\
4 & 73 & 65 & 45 & 24 & 21 \\
5 & 76 & 70 & 46 & 29 & 22 \\
6 & 79 & 74 & 47 & 33 & 22 \\
7 & 80 & 75 & 49 & 36 & 23 \\
8 & 81 & 75 & 51 & 38 & 24 \\
9 & 83 & 77 & 53 & 40 & 24 \\
10 & 85 & 79 & 55 & 41 & 25 \\
11 & 85 & 80 & 58 & 41 & 25 \\
12 & 86 & 82 & 66 & 41 & 25 \\
24 & 93 & 90 & 66 & 41 & 25 \\
\hline
\end{tabular}


Tabla 5. Fórmulas de tendencias de la mortalidad de larvas de Anopheles benarrochi por acción del polvo de raíz de Lonchocarpus utilis en dos calidades de agua

\begin{tabular}{ll}
\hline Dosis $(\mathrm{g} / \mathrm{L})$ & Agua destilada \\
\cline { 2 - 2 } 6,25 & $\mathrm{y}=8,4585 \operatorname{Ln}(\mathrm{x})+4,7324$ \\
3,1 & $\mathrm{y}=7,421 \operatorname{Ln}(\mathrm{x})+4,5754$ \\
2,1 & $\mathrm{y}=5,8474 \operatorname{Ln}(\mathrm{x})+3,6449$ \\
1 & $\mathrm{y}=4,1113 \operatorname{Ln}(\mathrm{x})+0,6815$ \\
0,15 & $\mathrm{y}=4,5621 \operatorname{Ln}(\mathrm{x})-0,7089$ \\
& Agua de criadero \\
\cline { 2 - 2 } 6,25 & $\mathrm{y}=7,9005 \operatorname{Ln}(\mathrm{x})+3.1769$ \\
3,1 & $\mathrm{y}=8,0049 \operatorname{Ln}(\mathrm{x})+1,4901$ \\
2,1 & $\mathrm{y}=5,3779 \operatorname{Ln}(\mathrm{x})+1,7041$ \\
1 & $\mathrm{y}=4,243 \operatorname{Ln}(\mathrm{x})-0,2775$ \\
0,15 & $\mathrm{y}=2,4012 \operatorname{Ln}(\mathrm{x})+0,5762$ \\
\hline
\end{tabular}

En agua de criadero, con la dosis $6,5 \mathrm{~g} / \mathrm{L}$ se observó mortalidad superior al 50\% de larvas tratadas a las 2 horas post-tratamiento; con la dosis $3,1 \mathrm{~g} / \mathrm{L}$ a partir de las 3 horas y con la dosis $2,1 \mathrm{~g} / \mathrm{L}$ a partir de las 8 horas post-aplicación se registró la misma eficacia. Con las dosis de 1,0 y $0,1 \mathrm{~g} / \mathrm{L}$ no se registraron valores de mortalidad superiores al $50 \%$ post-aplicación durante el desarrollo de los bioensayos.

Al observar el comportamiento de la mortalidad en el tiempo por dosis aplicada según el modelo de regresión lineal simple, se determinó que existían en algunos casos donde la tendencia era bastante similar pero con promedios diferentes con lo cual implicaría que

Tabla 6. Valores de dosis letales del polvo de raíz de Lonchocarpus utilis. en agua destilada según análisis probit a 6 horas de exposición

\begin{tabular}{cccc}
\hline \multicolumn{2}{c}{$\begin{array}{c}\text { Valor estimado } \\
\text { concentración g/L }\end{array}$} & \multicolumn{2}{c}{$\begin{array}{c}\text { 95\% Límites de } \\
\text { Confianza }\end{array}$} \\
\cline { 3 - 4 } Puntos & & Inferior & Superior \\
\hline DL1 & 0,0028 & 0 & 0,0197 \\
DL5 & 0,0137 & 0,0003 & 0,0602 \\
DL10 & 0,032 & 0,0015 & 0,1099 \\
DL15 & 0,0566 & 0,0041 & 0,1658 \\
DL50 & 0,6307 & 0,2504 & 1,1017 \\
DL85 & 7,0302 & 3,5035 & 31,6067 \\
DL90 & 12,4370 & 5,4281 & 84,2546 \\
DL95 & 28,9594 & 10,1231 & 369,4516 \\
DL99 & 141,3425 & 31,4873 & $6.115,9102$ \\
\hline
\end{tabular}

DL50 $=0,6307 \mathrm{~g} / \mathrm{L}$ de extracto crudo

DL90 $=12,4370 \mathrm{~g} / \mathrm{L}$ de extracto crudo. las dosis probadas tienen el mismo comportamiento, tal es el caso del tratamiento en agua destilada con las dosis 1,0 y $0,1 \mathrm{~g} / \mathrm{L}$ cuyas pendientes son 4,1 y 4,56 respectivamente; En agua de criadero con las dosis 6,25 y $3,1 \mathrm{~g} / \mathrm{L}$ las pendientes son 7,9 y 8,0.

En otros casos, las tendencias son diferentes lo cual evidencia que tienen comportamientos desiguales donde aquellas dosis con mayor pendiente implican que a menor concentración mayor mortalidad; tales son los casos de las dosis 6,$25 ; 3,1$ y $2,1 \mathrm{~g} / \mathrm{L}$ en agua destilada, donde las pendientes son 8,$45 ; 7,42$ y 5,8 respectivamente; asimismo con las dosis 2,$1 ; 1,0$ y $0,1 \mathrm{~g} / \mathrm{L}$, las pendientes son 5,37 ; 4,2 y 2,1 respectivamente.

Si comparamos el comportamiento de las mortalidades en ambos tipos de aguas, observamos tendencias como 8,43 y 7,4 en agua destilada y 7,9 y 8.0 en agua de criadero, por lo que utilizando dosis mayores de $3,1 \mathrm{~g} / \mathrm{L}$ se obtendrán mortalidades mayores del $80 \%$ a las 12 horas y mayor al $90 \%$ a las 24 horas post-tratamiento respectivamente (Tabla 5).

La susceptibilidad, (DL50 a las 6 horas) de las larvas de $A$. benarrochi a L. utilis en agua destilada fue $0,6307 \mathrm{~g} / \mathrm{L}$ y la DL90 fue 12,4370 $\mathrm{g} / \mathrm{L}$ (Tabla 6). A las 12 horas de exposición la DL50 fue 0,4824 g/L y la DL90 de 7,2314 g/ $\mathrm{L}$ del polvo de raíz respectivamente (Tabla 7).

Tabla 7. Valores de dosis letales del polvo de raíz de Lonchocarpus utilis. en agua destilada según análisis probit a 12 horas de exposición

\begin{tabular}{cccc}
\hline & $\begin{array}{c}\text { Valor estimado } \\
\text { concentración g/L }\end{array}$ & \multicolumn{2}{c}{$\begin{array}{c}\text { 95\% Límites } \\
\text { de Confianza }\end{array}$} \\
\cline { 2 - 4 } Puntos & & Inferior Superior \\
\hline DL 1 & 0,0035 & $* * * *$ & $* * * * *$ \\
DL 5 & 0,0149 & $* * * * *$ & $* * * * *$ \\
DL 10 & 0,0322 & $* * * * *$ & $* * * * *$ \\
DL 15 & 0,054 & $* * * * *$ & $* * * * *$ \\
DL 50 & 0,4824 & $* * * * *$ & $* * * * *$ \\
DL 85 & 4,3078 & $* * * * *$ & $* * * * *$ \\
DL 90 & 7,2314 & $* * * * *$ & $* * * * *$ \\
DL 95 & 15,5789 & $* * * * *$ & $* * * * *$ \\
DL 99 & 65,7195 & & \\
\hline
\end{tabular}

DL50 $=0,4824 \mathrm{~g} / \mathrm{L}$ de extracto crudo

$\mathrm{DL} 90=7,2314 \mathrm{~g} / \mathrm{L}$ de extracto crudo 
Cuando se usó agua de criadero, a las 6 horas los índices de susceptibilidad fueron la DL50= 1,3641 g/L y la DL90=27,576 g/L del polvo de raíz (Tabla 8). A las 12 horas de exposición los índices de la DL50=0,8342 $\mathrm{g} / \mathrm{L}$ y de la DL90= 9,8316 g/L de polvo de raíz L. utilis (Tabla 9).

Se observó que la tolerancia de las larvas de $A$. benarrochi a las dosis de L. utilis, se va perdiendo conforme aumenta el tiempo de exposición y la toxicidad va aumentando. Entre las de 6 y 12 horas de exposición en agua destilada la DL50 disminuyó 1,3 veces y la DL90 1,7 veces, mientras en agua de criadero la DL50 disminuyó 1,6 veces y la DL90 2,8 veces.

Al comparar susceptibilidades entre ambos tipos de tratamientos, se observó que en agua de criadero a las 6 horas post-tratamiento la toxicidad es 2,16 veces menos tóxica para valores de la DL50 y 2,23 veces menos tóxicas para valores de la DL90. A las 12 horas es 1,7 veces y la DL90 es 1,36 veces menos tóxica.

\section{Análisis estadístico}

L. utilis, indujo mortalidad en larvas de $A$. benarrochi, obteniéndose diferencias significativas entre los grupos tratados y el control $(\mathrm{p}=$ 0,000 para agua destilada y $p=0,000$ para agua de criadero). Para comprobar si la calidad del agua influye en la mortalidad, se realizó un MANOVA, obteniéndose un $\mathrm{p}=0,000$ como

Tabla 8. Valores de dosis letales del polvo de raíz de Lonchocarpus utilis en agua de criadero según análisis probit a 6 horas de exposición

\begin{tabular}{cccc}
\hline & $\begin{array}{c}\text { Valor estimado } \\
\text { concentración }\end{array}$ & \multicolumn{2}{c}{$\begin{array}{c}\text { 95\% Límites de } \\
\text { Confianza }\end{array}$} \\
\cline { 3 - 4 } Puntos & $\mathbf{g} / \mathbf{L}$ & Inferior & Superior \\
\hline DL 1 & 0,0058 & 0 & 0,0355 \\
DL 5 & 0,0285 & 0,0009 & 0,1088 \\
DL 10 & 0,067 & 0,0042 & 0,1994 \\
DL 15 & 0,1193 & 0,0119 & 0,3022 \\
DL 50 & 1,3641 & 0,7099 & 2,4374 \\
DL 85 & 15,5966 & 6,6220 & 125,2069 \\
DL 90 & 27,7576 & 10,0793 & 354,2969 \\
DL 95 & 65,2100 & 18,5364 & $1.676,4827$ \\
DL 99 & 323,6157 & 56,9819 & 31.553 .168 \\
\hline
\end{tabular}

DL50 $=1,3641 \mathrm{~g} / \mathrm{L}$ de extracto crudo

DL90 $=27,7576 \mathrm{~g} / \mathrm{L}$ de extracto crudo resultado de la prueba, por lo cual la hipótesis nula en la cual se planteaba que no existen diferencias significativas de mortalidad en ambos tipos de agua se rechazó. En consecuencia existe evidencia de al menos una dosis para afirmar que la calidad de agua influye en la mortalidad.

Al aplicarse las dosis polvo de raíz de $L$. utilis de forma individual se encontraron diferencias estadísticas significativas en ambos tipo de agua para la dosis de $0,15 \mathrm{~g} / \mathrm{L}(\mathrm{p}=0,021)$, sin embargo cuando se usa cualquiera de las otras dosis, estadísticamente producen el mismo resultado, no difieren significativamente entre ellas $(p=0,610 ; p=0,120 ; p=0,368 ; p=0,315)$.

Como el número de replicas es muy pequeño, solo 7 y no conociéndose la función de distribución de probabilidad de esta muestra se usó la pruebas no paramétrica de Kruskal Wallis, para la comparación entre dosis con el objetivo de establecer si existen diferencias estadísticas significativas. Se pudo observar que con agua destilada existen diferencias entre la mortalidad de las dosis $6,25 \mathrm{~g} / \mathrm{L}$ y las dosis 2,1; 1,0 y 0,15 g/L. Entre la dosis 3,1 y las dosis 2,1; 1,0 y 0,15 g/L. Entre las dosis 2,1 $\mathrm{g} / \mathrm{L}$ y las dosis 1,0 y $0,15 \mathrm{~g} / \mathrm{L}$; pequeñas diferencias entre las dosis 6,25 y $3,1 \mathrm{~g} / \mathrm{L}$ y ninguna diferencia entre las dosis $1,0 \mathrm{~g} / \mathrm{L}$ y $0,15 \mathrm{~g} / \mathrm{L}$. Asimismo, existen diferencias en la mortalidad del tratamiento en agua de criadero entre las dosis 6,25

Tabla 9. Valores de dosis letales del polvo de raíz de Lonchocarpus utilis en agua de criadero según análisis probit a 12 horas de exposición

\begin{tabular}{cccc}
\hline \multicolumn{2}{c}{$\begin{array}{c}\text { Valor estimado } \\
\text { concentración }\end{array}$} & \multicolumn{2}{c}{$\begin{array}{c}\text { 95\% Límites de } \\
\text { Confianza }\end{array}$} \\
\cline { 3 - 4 } Puntos & $\mathrm{g} / \mathrm{L}$ & Inferior & Superior \\
\hline DL 1 & 0,0095 & 0,0004 & 0,0403 \\
DL 5 & 0,0351 & 0,0032 & 0,1059 \\
DL 10 & 0,0707 & 0,0098 & 0,1782 \\
DL 15 & 0,1134 & 0,0209 & 0,2544 \\
DL 50 & 0,8342 & 0,4361 & 1,3267 \\
DL 85 & 6,1360 & 3,4452 & 18,2848 \\
DL 90 & 9,8381 & 5,0153 & 38,1007 \\
DL 95 & 19,8013 & 8,5783 & 115,3068 \\
DL 90 & 73,5293 & 22,8218 & 946,5381 \\
\hline DL50 $=0,8342 \mathrm{~g} / \mathrm{L}$ de extracto crudo \\
DL90 $=9,8316 \mathrm{~g} / \mathrm{L}$ de extracto crudo
\end{tabular}

Rev. peru. biol. 11(1): 87- 94 (2004) 
y las dosis 2,$1 ; 1,0$ y $0,15 \mathrm{~g} / \mathrm{L}$. Entre la dosis 3,1 y las dosis 2,1; 1,0 y $0,15 \mathrm{~g} / \mathrm{L}$. Entre las dosis 2,1 y las dosis 1,0 y $0,15 \mathrm{~g} / \mathrm{L}$, y entre las dosis $1,0 \mathrm{y}$ $0,15 \mathrm{~g} / \mathrm{L}$; casi ninguna diferencia entre los tratamientos con las dosis 6,25 y $3,1 \mathrm{~g} / \mathrm{L}$.

\section{Discusión y conclusiones}

Los trabajos realizados en el Perú por Wille (1952), Cisneros y Fukuda (1965), Beingolea (1988), Vílchez y Sánchez (1993), Zúñiga (1993), Vílchez (1994) y Ruiz (1995), reportaron efectividad de $L$. utilis contra plagas de hortalizas, pulgas de algodonero, mosca blanca de los cítricos, cigarritas, Heliothis sp. plagas de col y plagas de cultivos de maíz.

Las primeras aplicaciones del Lonchocarpus $\mathrm{sp}$ «barbasco» $\mathrm{o}$ «cube» sobre larvas de mosquitos no reportaron efectividad en el control de éstas. Gibson (1928), solo encontró efectividad sobre peces a los 15 minutos de aplicación de extractos de cube, pero no demostró efectividad sobre larvas de Culex sp. y Anopheles sp. Similares resultados reporta Wille (1939) al utilizar extractos de «cube» con 5\% de rotenona, contra larvas de $A$. pseudopunctipennis, después de 15 horas de tratamiento.

El presente estudio demuestra la eficacia de polvo de raíz de L. utilis para controlar larvas de $A$. benarrochi, con dosis de 3,1 g/L. Similar eficacia obtuvo Gamarra (1940), quien utilizó extractos de raíz de L. nicou contra larvas de Anopheles sp. y Culex sp, reportando $100 \%$ de mortalidad, con dosis hasta 8 veces mayor a la reportada en el presente trabajo. No se ha encontrado referencias recientes del uso de los extractos Lonchocarpus sp como insecticida en salud publica.

La eficacia del polvo de raíz de L. utilis como biocida, es similar a la de otros extractos de plantas contra larvas de Anopheles sp. Así Dharmashaktu et al. (1987) utilizaron extractos de hojas de Agave americana, Mwangi y Mukiama (1988) usaron fracciones de granos de fruto de Melia volkensii, Saxena et al (1993) emplearon alcaloides de Annona squamosa y Perich et al. (1994) usaron extractos de flores de Tagetes minuta sobre larvas de Culex sp., Jackson et al. (1990) utilizaron semillas de Sorghum bicolor; Sherif et al. (1985) emplearon extractos crudos y homogenizados de Elodea nuttalli. Los trabajos realizados indican que la variación de los resultados, se atribuyen a factores como la cepa, edad y vigor de las larvas; concentración del principio activo, tipo de extracto empleado, ya que no todos emplearon los mismos protocolos en el momento de evaluar la toxicidad de los extractos.

Estos primeros resultados muestran que el uso del polvo de raíz de L. utilis en el control de $A$. benarrochi, podría constituir una importante alternativa de uso en regiones económicamente pobres y de difícil acceso por ser eficaz, de fácil aplicación y de bajo costo para controlar la malaria mediante la eliminación del vector en los casos de brotes epidémicos y sólo en criaderos temporales, debido a que su efecto biocida no es selectivo con otros organismos de la fauna acuática.

Se ha observado mayor eficacia con la dosis 3,1 g/L del polvo de raíz de L. utilis sobre larvas de $A$. benarrochi, obteniéndose mortalidades mayores al $80 \%$ a las 12 horas y del $90 \%$ a las 24 horas de aplicación. Se evidenció que existe mayor eficacia con el tratamiento utilizando agua destilada que con el agua de criadero.

\section{Literatura citada}

Beingolea 0. 1988. Protección Vegetal. Edit. Banco Agrario, Lima. Perú. pp 30.

Calderón, F. G, Fernández L., y Valle T., J. 1995. Especies de la fauna anophelina, en su distribución y algunas consideraciones sobre su abundancia, infectividad e importancia en la transmisión de la Malaria en el Perú. Rev. Per. de Epid. 8(1):5-53.

CDPI-CIP. 1992. Centro de Desarrollo Profesional en Ingeniería (CDPI). Colegio de Ingenieros del Perú (CIP). En: Rotenona: Un Insecticida Orgánico de Origen Vegetal Compatible con el Medio Ambiente. Rev. Agro Enfoque. 55: 23.

Cisneros F., y Fukuda 0. 1965. Efectos de Mezclas de Rotenona y Aceites emulsionables contra la mosca blanca de cítricos Aleurotrixus floccosus Rev. Per. Ent. 8(1): 76-80.

Consoli, R, y De Oliveira, R. 1994. Principais mosquitoes de importancia sanitaria no Brasil. Edit. Fiocruz. Río de Janeiro. 225 pp. 
Dharmashaktu, N. S., Prabhakaran, P. K., and Menon, P. K. 1987. Laboratory study on the mosquito larvicidal propierties of leaf and seed extract of the plant Agave americana. J. Trop. Med. Hyg. 90(2):79-82.

Gamarra Caller, Gonzalo. 1940. El empleo del Barbasco como larvicida contra el Paludismo y otras enfermedades transmitidos por los Culicideos. Rev. Sanid. Milit. Peru., 13: 103-114

Ghorham, I. R, Stojanovich C. J, y Scott H. G. 1973. Clave ilustrada para los mosquitos anophelinos de Sudamérica Occidental. Mosq. System. 5(2):97-154.

Gibson, A. 1928. Proceeding of the 15TH. Annual Meeting of the New Jersey. Mosquito Experimentation Association, Atlantic City. $136-146$ pp.

Jackson, F. L., Behkeit S, S., El Etr S. M., and Quach N. K. 1990. Larvicidal effects of grain sorghum (Shorgum bicolor) seedlin extracts upon Culex pipiens larvae. J. Am. Mosq. Control Assoc. 6(3):500- 503.

Lizárraga, T. A. 1992. Curso: Manejo Ecológico de Plagas. Chiclayo -Cajamarca. (Nov/Dic).

Lizárraga, T. A. 1993. Extractos vegetales para la Agricultura: Insecticidas de menor grado de toxicidad. El Comercio. Feb. 19 Rev. El Agro. N 9. Sección Consultorio.

Mwangi, R. W., and T. K. Mukiama. 1988. Evaluation of Melia volkensii extract fractions as mosquito larvicides. J. Am. Mosq. Control Assoc. 4(4):442-427.

Nongrados Córdova, Diana Doris. 1999. Evaluación de la actividad larvicida del Bacillus sphaericus 2362 sobre larvas de Anopheles pseudopunctipennis (Theobald) y Culex quinquefasciatus (Say) en bioensayos de laboratorio. Tesis para optar el Titulo de Biólogo, mención en Microbiología y Parasitología. Facultad de Ciencias Biológicas, UNMSM.

OMS. 1960. Resistencia a los insecticidas y lucha contra los vectores. Décimo lnforme Técnico del Comité de Expertos en Insecticidas. Organización Mundial de la Salud. Inf. Tec. 191: 26-31.

OMS. 1988. Clasificación de pesticidas según su grado de peligro, recomendada por la OMS. y guía para su clasificación. 1988-1989. Pesticide Development and Safe Use Unit Division of Vector, Biology and control (VBC). Organización Mundial de la Salud (OMS). Ginebra. WHO/VBC/88.953.

OMS. 1976. Resistencia de los vectores y reservorios de enfermedades a los plaguicidas. $22^{\circ} \ln$ forme Técnico del Comité de Expertos de la Organización Mundial de la Salud en Insecticidas. Serie de Informes Técnicos No 67939 pp.
Perich, M., Wells C., Bertsch W., and Tredway K. E. 1994. Toxicity of extracts from three Tagetes against adults and larvae of yellow fever mosquito and Anopheles stephensi (Diptera: Culicidae). J. Med. Entomol. 31(6):833 -837.

Proyecto Vigía. 1999. Impacto económico de la Malaria en el Perú. Ministerio de Salud. Serie Vigía No 1. $127 \mathrm{pp}$.

RAA. 1993. I Taller Nacional: Plantas con propiedades biocidas al servicio del agricultor. Red de Acción en Alternativas al uso de Agroquímicos (RAAA). Diciembre.

Ruiz Beteta, Rosmery. 1995. Dosis de Aplicación del cube (Lonchocarpus utilis A. C. Smith) en el control de Gusano Cogollero Spodoptera frugiperda (I. E. Smith) en el cultivo de Maíz (Zea mays L.). Tesis Ingeniero Agrónomo. Facultad de Agronomía Universidad Nacional de San Martín.

Saxena, R. C., Harshan V., Saxena A., Sukumaran P., Sharma, M. C., and M. Lakshamana Kumar. 1993. Larvicidal and chemosterilant activity of Annona squamosa alkaloids against Anopheles stephensi. J. Am. Mosq. Control Assoc. 9(1):54 -87.

Sherif A, Hall R. G, and M. M. EI-Amamy. 1985. Effects of an Elodea extract on immature stages of Culex quinquefasciatus Say. J. Fla. Anti-Mosq. Assoc. 56:82-85.

Vílchez E. J, y Sánchez G. V. 1993. Uso de la Rotenona (Lonchocarpus nicou) para controlar plagas de la col en Lima. Rev. Per. Ent. 36:65-68

Vílchez, E. J. 1994. Efectividad y forma de aplicación de Rotenona (Lonchocarpus nicou L.) en el control de plagas de la Col (Brassica oleracea var. capitata). Tesis Ingeniero Agrónomo. Universidad Agraria la Molina (UNALM). Perú.

Wille, J. E., 1952. Entomología Agrícola del Perú. Ministerio de Agricultura, Instituto de Altos Estudios Agrícolas del Perú. Lima- Perú. 543 pp.

Wille, J. E., Ocampo, J. A., Weberbauer, A., y Schofield, D. 1939. El cube (Lonchocarpus utilis) y otros barbascos en el Perú. Boletín No.12. Ministerio de Fomento. Dirección de Agricultura, Ganadería y Colonización. Instituto de Altos Estudios Agrícolas del Perú. Lima - Perú. 117 pp.

Zúñiga, M. E. 1993. Efectos de la Rotenona a los controladores biológicos en el control de Hylemia sp en el cultivo de Maíz en la Sierra. En: I Seminario Taller Internacional: Aportes del Control Biológico en la Agricultura Sostenible. Mayo. $50 \mathrm{pp}$ 